\title{
WHY CHILDREN'S SUGGESTIBILITY REMAINS A SERIOUS CONCERN
}

\author{
AMYE R. WARREN* AND DOROTHY F. MARSIL**
}

INTRODUCTION

After more than two decades of continuous contemporary research on the child as a witness in legal proceedings, a great deal is known about children's eyewitness memory and suggestibility. Excellent reviews of this research are available, ${ }^{1}$ and their summaries and conclusions will not be reiterated here. Instead, this article will focus on six areas representing some of the most intractable problems that will require further attention from scientists and practitioners alike. This list of issues is selective and somewhat idiosyncratic, but should serve to illustrate why the current understanding of children's suggestibility is far from complete. Research on each issue will be highlighted, concentrating primarily on studies published or presented in the past ten years.

II

\section{SIX REMAINING ISSUES}

\section{A. Suggestibility is Not Limited to Preschool Children}

In the past ten years, research on children's capacities as witnesses has focused heavily on preschoolers, ${ }^{2}$ who are disproportionately susceptible to suggestion. ${ }^{3}$ The focus on preschoolers was based on both applied and theoretical grounds. For example, investigations in the McMartin Preschool case ${ }^{4}$ and State v. Michaels ${ }^{5}$ featured particularly problematic interviews with large numbers of very young children in day-care settings. Knowledge of cognitive development principles led child witness researchers to predict that preschoolers' relative

Copyright (C) 2002 by Amye R. Warren and Dorothy F. Marsil

This article is also available at http://www.law.duke.edu/journals/65LCPWarren.

* University of Chattanooga Foundation Professor of Psychology, University of Tennessee at Chattanooga. B.S. 1980, Georgia Institute of Technology; Ph.D. 1984, Georgia Institute of Technology.

** Ph.D. Candidate, University of Kentucky. B.A. 1995, University of Tennessee; M.S. 1999, University of Tennessee.

1. Stephen J. Ceci \& Maggie Bruck, The Suggestibility of the Child Witness: A Historical Review and Synthesis, 113 PSYCHOL. BULL. 403 (1993).

2. The term "preschooler" refers to children between the ages of three and five.

3. Maggie Bruck \& Stephen J. Ceci, The Suggestibility of Children's Memory, 50 ANN. REV. PSYCHOL. 419 (1999).

4. Satz v. Superior Court, 225 Cal. App. 3d 1525 (Cal. Ct. App. 1990).

5. 625 A.2d 489 (N.J. Super. Ct. App. Div. 1993). 
cognitive limitations could lead to greater susceptibility to suggestive influences and, in turn, to a variety of problems for their interviewers. For example, Nancy Perry and her colleagues demonstrated that preschoolers have difficulty determining whether they really understand complex questions and frequently inaccurately answer questions that they do not understand. ${ }^{6}$

Although the research attention devoted to preschoolers was certainly warranted, it has led to a relative neglect of the equally important population of older children. This trend, however, appears to be changing. In recent studies, researchers are increasingly including older children. ${ }^{7}$ The findings indicate that suggestibility generally declines over the school years but that even adolescents can be significantly more suggestible than adults. ${ }^{8}$ On the other hand, some studies demonstrate that, under certain conditions, older children and adults can be more suggestible than younger children. ${ }^{9}$ The following sections briefly review six studies that included children older than preschoolers.

Jennifer Ackil and Maria Zaragoza examined the suggestibility of first graders, third graders, fifth graders, and college students. ${ }^{10}$ The subjects viewed a brief video and then heard an experimenter read a summary of the video that included some misleading information. ${ }^{11}$ Either immediately afterwards or one week later, participants were given memory tests. ${ }^{12}$ Evidence of suggestibility was found for all age groups, but first graders were more susceptible to suggestion than third and fifth graders, who were in turn more susceptible to suggestion than college students. ${ }^{13}$ Essentially, the same pattern of age differences was found in the proportion of the suggested items that participants claimed to have actually seen in the video, as opposed to remembering from the summary. ${ }^{14}$

Julie Robinson and Pamela Briggs also showed a film to their participants, who were four- to five-year-olds, eight- to nine-year-olds, and adults. ${ }^{15}$ They

6. Nancy Perry et al., When Lawyers Question Children: Is Justice Served?, 19 LAw \& HuM. BEHAV. 609, 621-25 (1995).

7. Jennifer K. Ackil \& Maria S. Zaragoza, Developmental Differences in Eyewitness Suggestibility and Memory for Source, 60 J. EXPERIMENTAL CHILD PSYCHOL. 62 (1995); William Cassel et al., Developmental Patterns of Eyewitness Responses to Repeated and Increasingly Suggestive Questions, $61 \mathrm{~J}$. EXPERIMENTAL CHILD PSYCHOL. 116 (1996); Pamela Coxon \& Tim Valentine, The Effects of the Age of Eyewitness on the Accuracy and Suggestibility of their Testimony, 11 APPLIED COGNITIVE PSYCHOL. 415 (1997); Marc A. Lindberg et al., Eyewitness Testimony for Physical Abuse as Function of Personal Experience, Development, and Focus of Study, 21 J. APPLIED DEVELOPMENTAL PSYCHOL. 555 (2000) [hereinafter Lindberg, Eyewitness]; Marc Lindberg, An Interactive Approach to Assessing the Suggestibility and Testimony of Eyewitnesses, in THE SUGGESTIBILITY OF CHILDREN's RECOLLECTIONS 47, 49-52 (John Doris ed., 1991) [hereinafter Lindberg, Interactive]; Julie Robinson \& Pamela Briggs, Age Trends in Eyewitness Suggestibility and Compliance, 3 PsYCHOL. CRIME \& L. 187 (1997).

8. Ackil \& Zaragoza, supra note 7, at 74-75; Robinson \& Briggs, supra note 7, at 196-98; Cassel et al., supra note 7, at 123-24; Lindberg, Eyewitness, supra note 7, at 567-72; Lindberg, Interactive, supra note 7 , at $50-54$.

9. Lindberg, Interactive, supra note 7, at 50-54.

10. Ackil \& Zaragoza, supra note 7 , at 62.

11. Id. at 63 .

12. $I d$.

13. Id. at $67-72$.

14. Id. at 72-74.

15. Robinson \& Briggs, supra note 7, at 191-92. 
then asked a series of questions, some of which were misleading. ${ }^{16}$ A day later, they asked another set of questions. ${ }^{17}$ In terms of correct answers to misleading questions, the youngest children's performance was poorer than that of eight- to nine-year-olds and adults. The latter two groups, however, did not significantly differ. $^{18}$ In contrast, when suggested answers to misleading questions were examined separately, the study showed that both age groups of children were more suggestible than adults, but that the children did not differ from one another..$^{19}$

In a similar study, Pamela Coxon and Tim Valentine examined the relative suggestibility of seven- to nine-year-olds, young adults, and elderly adults. ${ }^{20}$ Participants viewed a film and later answered two sets of questions, some of which were misleading. ${ }^{21}$ Children were significantly more likely to be misled than were the two groups of adults. Again, the two adult groups did not differ from one another. ${ }^{22}$

William Cassel, Claudia Roebers, and David Bjorkland showed a film about a bicycle theft to kindergartners, second graders, fourth graders, and adults and then asked some of them misleading questions a week later. ${ }^{23}$ Kindergartners were more susceptible to suggestion than all older age groups, who did not significantly differ from one another, ${ }^{24}$ except when the suggestions concerned information that was central ${ }^{25}$ to the premise of the entire event. ${ }^{26}$ This suggests that the importance of the information sought is predictive of a child's ability to recall it and to resist misleading information about it. When the information is important, younger children may be more likely to recall it, and therefore their recall and ability to resist misleading questions may be equal to that of older children and even adults.

Marc Lindberg, John Keiffer, and Stuart Thomas compared the suggestibility of nine-year-olds, thirteen-year-olds, and seventeen-year-olds. ${ }^{27}$ All children watched a videotape that included an apparent act of physical abuse by a mother against her son. ${ }^{28}$ Later they were given two memory tests about the

16. Misleading questions are those that incorporate or suggest inaccurate information that may lead to incorrect answers. For example, if a man in the film had a mustache but no beard, a misleading question might ask, "What color was the man's beard?" Id. at 192.

17. $I d$.

18. Id. at 193 .

19. Id. at 194-96.

20. Coxon \& Valentine, supra note 7, at 420-21.

21. Id.

22. Id. at 425 .

23. Cassel et al., supra note 7, at 121-22.

24. Id. at 123-27.

25. For example, a question concerning a central detail might be whether the thief asked permission to take the bike, whereas a question concerning a peripheral detail might ask about the color of a bystander's shirt.

26. Id. at 128 .

27. Lindberg, Eyewitness, supra note 7, at 561.

28. Id. 
film, the first of which included misleading questions. ${ }^{29}$ The nine-year-olds were significantly more suggestible than were the thirteen- and seventeen-year-olds about central details of the film, including how many times the mother hit the boy and how much blood came from his nose..$^{30}$ Additionally, the youngest children were much more likely than the older two groups to fabricate responses to nonleading questions for which they could not recall the answers. ${ }^{31}$ Even the seventeen-year-olds, however, were suggestible, and a third of them confabulated answers. ${ }^{32}$

Finally, another study by Marc Lindberg revealed circumstances in which older children and adults can be more suggestible than younger children. ${ }^{33}$ In this study, third graders, sixth graders, and college students were tested on their memories for a film about students taking a test. ${ }^{34}$ Prior to the film, some of the participants were misled to believe that the students would cheat. ${ }^{35}$ Half of the participants were misled about cheating through questions that followed the film. $^{36}$ Although in general the younger children were more suggestible than the older children and college students, the opposite finding occurred for one question involving a student in the film who had asked about the time of day. ${ }^{37}$ Apparently the older children and college students, who had more extensive knowledge of cheating, assumed that asking about the time of day was a sophisticated cheating strategy, when in fact it was an innocent question. ${ }^{38}$

Despite the progress in understanding suggestibility over the entire range of childhood, research has not yet produced equivalent levels of knowledge about older children, preschoolers, and adults. Increasing age typically is associated with increased resistance to suggestion and increased strength of memory. ${ }^{39}$ On the other hand, increasing age also brings with it changes that can weaken the accuracy of eyewitness testimony, such as greater skill in deception. ${ }^{40}$ In some studies, suggestibility appears to decrease in a gradual linear fashion with increasing age, whereas in other studies, older children appear to have reached the asymptote, displaying adult levels of suggestibility. ${ }^{41}$ More research is necessary to determine the conditions under which and the age at which children show equivalent suggestibility to adults. Moreover, the level of ecological va-

29. Id. at 562 .

30. Id. at 564 .

31. Id. at 567 .

32. Id. at 567-70.

33. Lindberg, Interactive, supra note 7, at 54.

34. Id. at 49 .

35. Id. at 49-50.

36. $I d$.

37. Id. at 51-52.

38. Id. at 52-53.

39. Ceci \& Bruck, supra note 1 , at 421 .

40. Id. at 426-27.

41. Id. at 431-34. 
lidity ${ }^{42}$ in research with older children requires improvement in that most such studies have not used participatory events. Finding events that work equally well across a wide age range is difficult, but it is a goal we should strive for in future research.

\section{B. Suggestiveness is Not Limited to Leading Questions}

The majority of studies on children's suggestibility has focused on children's responses to leading and misleading questions. A vast literature now exists on different types of questions and the effects that they appear to have on the accuracy and completeness of children's testimony. ${ }^{43}$ Suggestion, however, takes on many different forms. ${ }^{44}$ As with the other topics reviewed, the discussion that follows will be limited to a few recent studies that illustrate some of these lesser-studied forms of suggestion.

Sena Garven and her colleagues recently published two studies on the effects of potentially suggestive techniques used by interviewers in the McMartin Preschool case. ${ }^{45}$ In their first study, three- to six-year-olds were interviewed about a prior event with either suggestive questions only or a package of inappropriate interview techniques, including suggestive questions and three additional factors they termed social influence, reinforcement, and invitations to speculate. ${ }^{46}$ Social influence involved giving information about the event that other children ("big kids") had supposedly already provided. ${ }^{47}$ Reinforcement involved praising the children's memory and intelligence if they answered "yes" to leading and misleading questions, or exhorting them to try harder or expressing mild disapproval if they answered "no." Children were invited to speculate by being asked to think hard and then being asked a leading or misleading question beginning with: "Do you think maybe that $\mathrm{X}$ happened?" Children in this package condition were significantly more likely to answer misleading questions with the suggested information than were children only asked suggestive questions, and this was equally true across the age range. ${ }^{50}$

42. Ecological validity refers to the extent to which a study is representative of the "real-world" situation to which it was designed to apply. Given that the crime of child abuse is the event to which most studies of children's suggestibility attempt to relate, then events in which children are interactive participants, such as playing a game or undergoing a physical examination, rather than mere bystanders, such as watching a videotaped crime, are likely to be more valid.

43. See generally Debra A. Poole \& Michael E. LAmb, Investigative Interviews of CHILDREN: A GUIDE FOR HELPING PROFESSIONALS (1998).

44. For a comprehensive review, see Stephen J. Ceci et al., The Suggestibility of Children's Testimony, in FALSE-MEMORY CREATION IN CHILDREN AND ADULTS 169 (David Bjorkland ed., 2000).

45. Sena Garven et al., More than Suggestion: The Effect of Interviewing Techniques from the McMartin Preschool Case, 83 J. APPLIED PSYCHOL. 347, 350-54 (1998) [hereinafter Garven, Suggestion]; Sena Garven et al., Allegations of Wrongdoing: The Effects of Reinforcement on Children's Mundane and Fantastic Claims, 85 J. APPLIED PSYCHOL. 38, 41-45 (2000) [hereinafter Garven, Allegations].

46. Garven, Suggestion, supra note 45 , at 350.

47. Id. at 351 .

48. Id.

49. Id.

50. Id. at 352 . 
Most of the misleading suggestions implied wrongdoing on the part of a male experimenter. $^{51}$ Children given the whole package of suggestive techniques made false allegations over three times more often than did children only asked suggestive questions. ${ }^{52}$

In their second study, Garven and her colleagues focused on the effects of reinforcement and the provision of event information supposedly obtained from other children, called co-witness information, on children's tendencies to accept false suggestions regarding actions of a male experimenter that were both mundane, such as tearing a book, and fantastic, such as taking the child on a helicopter ride. ${ }^{53}$ In this study of five- to seven-year-olds, children who received cowitness information made more mundane false allegations than did the children in the control group, ${ }^{54}$ but did not differ in terms of fantastic false allegations. ${ }^{55}$ In contrast, reinforcement had a strong impact on children's answers to both types of misleading questions. ${ }^{56}$ Thirty-five percent of children who received reinforcement made false mundane allegations, compared to thirteen percent for the controls, and fifty-two percent of the same children made false fantastic allegations, compared to only five percent for controls. ${ }^{57}$ In addition, false allegations tended to carry over to a later interview-two to three weeks afterwards-even when no reinforcement was used at that time..$^{58}$

Another set of studies has examined the effect of interviewers' distortions or misinterpretations of children's answers. ${ }^{59}$ For example, in one interview, a twoyear-old child said, "G.A. touched me." ${ }^{6}$ Her interviewer responded, "Jesus loves me? Is that what you said?"; the child replied, "Yeah."

Kim Roberts and Michael Lamb analyzed a sample of sixty-eight sexual abuse interviews involving children between the ages of three and fourteen years, and found 140 instances of interviewer distortions and misinterpretations. $^{62}$ Many of these distortions involved the identities of people being discussed in the interview, and many others involved actions. ${ }^{63}$ Children actually

51. Id.

52. Fifty-eight percent versus seventeen percent. $I d$.

53. Garven, Allegations, supra note 45, at 38-39.

54. Twenty-nine percent versus nineteen percent. Id. at 42 .

55. Id. at $42-43$.

56. Id.

57. $I d$. at 42 .

58. Id. at 44-45.

59. Kim Roberts \& Michael E. Lamb, Children's Responses when Interviewers Distort Details During Investigative Interviews, 4 LEGAL \& CRIMINOLOGICAL PSYCHOL. 23, 23-31 (1999); Amye R. Warren et al., "It Sounds Good in Theory, But ...": Do Investigative Interviewers Follow Guidelines Based on Memory Research?, 1 CHILD MALTREATMENT 231, 231-45 (1996) [hereinafter Warren et al., Sounds]; Jennifer S. Hunt \& Eugene Borgida, Developmental Differences in Witnesses' Responses to Modifications, Eliciting Accurate Testimony from Children: What Works, What Doesn't, and Why?, A Symposium presented at the Biennial Meeting of the Society for Research in Child Development, Amye R. Warren \& Debra A. Poole, Chairs (April 1999).

60. Warren, Sounds, supra note 59, at 235.

61. Id.

62. Roberts \& Lamb, supra note 59, at 23-25.

63. Id. at 29 . 
agreed with the distortions one-third of the time, and explicitly corrected or disagreed with only one-third of them. ${ }^{64}$ In the absence of corrective feedback, the interviewers continued to use the distorted version of the information during the remainder of the interviews. ${ }^{65}$ However, the children later mentioned the distorted information only rarely, and their tendency to do so was unrelated to their initial agreement or disagreement. ${ }^{66}$

In actual interviews, it is impossible to determine whether interviewers' distortions are minor or major errors relative to the reality of the original events. ${ }^{67}$ Furthermore, children may fail to disagree with interviewer distortions because the interviewers' interpretations may in fact be correct. ${ }^{68}$ Therefore, Jennifer Hunt and Eugene Borgida designed an experiment in which four- to five-yearolds, nine- to eleven-year-olds, and adults witnessed a videotaped event and then were later interviewed. ${ }^{69}$ The interviewers were instructed to distort five of the answers. Overall, participants disagreed with only twenty-two percent of the modifications, and adults were significantly more likely than children to disagree. ${ }^{70}$ In an unbiased follow-up interview, the younger children-four- to fiveyear-olds-were more likely to incorporate prior interviewer distortions into their answers. ${ }^{71}$ Interestingly, information contained in interviewer distortions that were corrected-that is, explicitly disagreed with-was never included in subsequent reports. $^{72}$ In other words, when children clearly and confidently recalled information well enough to disagree with their interviewers, the interviewers' suggested misinformation was never mentioned in the children's later descriptions or answers to questions about events.

Research has only recently begun to examine alternate forms of suggestion, some of which are explicit and obviously coercive, while others may be subtle and quite unintentional, such as distortions. Although much more research on these types of suggestion is required, it is clear that suggestions may be equally or even more detrimental to children's testimonial veracity than leading questions. Just as with the research on leading questions, the controversy over these other suggestive techniques is likely to evolve over time, from whether they promote false allegations to how often they are used in typical interviews. ${ }^{73}$

64. Id. at $27-28$.

65. Id. at 29 .

66. Hunt \& Borgida, supra note 59.

67. Id.

68. $I d$.

69. $I d$.

70. $I d$.

71. $I d$.

72. $I d$.

73. For a discussion of the argument over the prevalence of leading questions in typical interviews, see Thomas D. Lyon, The New Wave in Children's Suggestibility Research: A Critique, 84 CORNELL L. REV. 1004, 1033-37 (1999), and the rebuttal by Stephen J. Ceci \& Richard D. Friedman, The Suggestibility of Children: Scientific Research and Legal Implications, 86 CORNELL L. REV. 33, 63-70 (2000). 


\section{Suggestibility is Not Confined to Formal Interviews}

Most of the research on the suggestibility of child witnesses has focused on fairly explicit suggestions made in the course of formal interviews with children about prior events. ${ }^{74}$ This is understandable, given that one can better document and control the formal interview process. Much of children's exposure to suggestive influences, however, probably occurs outside the formal interview setting. ${ }^{75}$ Suggestions may be made by parents, other adults, or other children prior to the first formal investigative interview or between repeated forensic or clinical interviews. ${ }^{76}$

Such outside suggestive influences have only recently begun to receive research attention. Debra Poole and Stephen Lindsay designed the innovative procedure of having parents provide misleading information to their children prior to two neutral, nonleading interviews. ${ }^{77}$ Children, ages three to eight years, participated in an experimental session with Mr. Science, who conducted some science demonstrations. ${ }^{78}$ Immediately afterward, they were neutrally interviewed. ${ }^{79}$ Approximately three months later, parents were sent books to read to these children at home. ${ }^{80}$ The books were about a visit to Mr. Science and described events that had occurred during the child's own visit, as well as events that had not occurred. ${ }^{81}$ Although the children were not told that the events in the story accurately described their own visits to Mr. Science, they also were not told that the stories contained false information. ${ }^{82}$ Within a few days after parents read the stories, and then again after a month-long delay, children were interviewed neutrally, with the interviews progressing from free recall to direct, yes or no questions about experienced (true) and nonexperienced (false) events. $^{83}$ The key findings of this study were that twenty-one percent of the events reported in free recall three and one-half months after the event, and ten percent four and one-half months after the event, were false because they had not been actually experienced. ${ }^{84}$ The rates of reporting false information in free recall did not differ by age across this range from three to eight years. ${ }^{85}$ More than one-third of the children falsely answered direct questions about nonexperienced touching after three and one-half months. By four and one-half months, older children's false acquiescence dropped, as it had been at least a

74. Debra A. Poole \& D. Stephen Lindsay, Children's Eyewitness Reports after Exposure to Misinformation from Parents, 7 J. EXPERIMENTAL PSYCHOL.: APPLIED 27 (2001) [hereinafter Poole, Eyewitness].

75. Id. at 27-28.

76. Id.

77. Id. at 28-29.

78. Id. at 29.

79. $I d$.

80. Id. at 30 .

81. $I d$.

82. $I d$.

83. Id. at 30-31.

84. Id. at 33 .

85. Id. at 34 . 
month since they were exposed to the misinformation, but three- to four-yearolds' false acquiescence increased, such that over half of them reported false information about nonexperienced touching. ${ }^{86}$ These findings raise concerns about the potential of false information to carry over into children's responses during later nonleading interviews.

Michelle Leichtman and Stephen Ceci took another approach to supplying preschoolers with misleading information outside of the interview context. ${ }^{87}$ Prior to a preschool visit by a man called Sam Stone, children were presented negative stereotypes of him. ${ }^{88}$ Sam was depicted as clumsy and prone to breaking objects. ${ }^{89}$ Children in this "stereotype only" condition then experienced Sam's visit, during which he did not break or harm anything, and were neutrally questioned once a week for the next four weeks about the visit. ${ }^{90}$ In a fifth and final, neutral interview, more than one-third of children responded falsely to a direct question about Sam Stone's behavior, such as "Did he rip the book?," in a direction consistent with the negative stereotype, despite the fact that these children had not been asked any misleading questions. ${ }^{91}$

Similarly, in the Lindberg study described above, prior to viewing a video depicting a mother hitting her son, grade schoolers and college students were given one of three descriptions of the video. ${ }^{92}$ In one, they were told that the mother was mean and had been previously arrested for child abuse ("mean mom"); in the second, they were told that the son's behavior had been very problematic at home and school, and that the mother had already tried many gentle forms of discipline ("bad boy"); and in the third, they were given no biasing information (neutral). ${ }^{93}$ The form of instructions affected many of the responses to both suggestive and nonsuggestive questions. ${ }^{94}$ For example, when asked how hard the boy deserved to be hit, those in the bad boy condition thought he should have been hit harder than did those in the neutral condition, who in turn thought he should be hit harder than did those in the mean mom condition. ${ }^{95}$

Even when false information is never presented to children, whether within or outside of interview questions, false reports may emerge nonetheless. When children's memories become distorted without external influence, it is sometimes termed "autosuggestibility." In one form of autosuggestibility, children

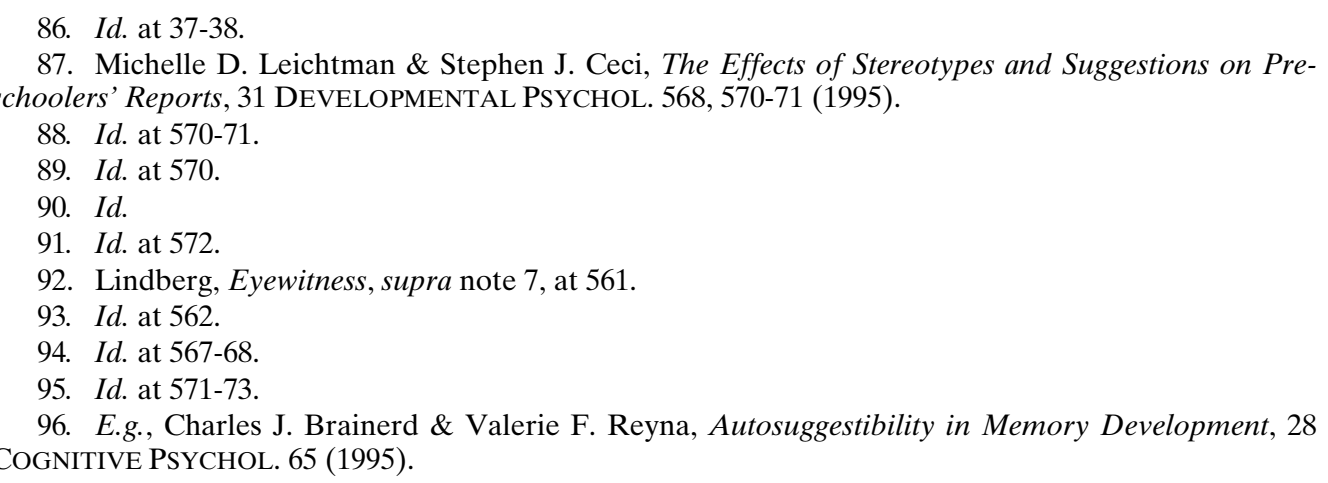


and adults may distort information to make it more congruent with their prior knowledge. ${ }^{97}$ In the Lindberg study, older children and adults were more likely to answer a nonleading question with false information than were younger children. ${ }^{98}$ The younger children were not as suggestible because they did not possess sophisticated, elaborate knowledge of cheating. ${ }^{99}$ In another example of autosuggestion due to prior knowledge, Stephen Ceci and his colleagues presented a tape-recorded story to seven- and ten-year-olds. ${ }^{100}$ The stories featured familiar or unfamiliar television characters; in some versions, the familiar characters were described in ways that clashed with their usual portrayals, such as a superhero who was too weak to carry a can of paint. ${ }^{101}$ Children were asked to rate the characters and their behavior either immediately after the story or three weeks later. ${ }^{102}$ When asked immediately, children correctly recalled the character descriptions and behavior, but three weeks later, children's ratings were distorted in the direction of their prior knowledge of the characters' typical attributes and behaviors. ${ }^{103}$ On the other hand, when story characters were completely unfamiliar, these types of distortions did not occur even after the delay. ${ }^{104}$ Therefore, Ceci and colleagues concluded that when information conflicts with prior knowledge, prior knowledge over time will distort or perhaps even supplant that information. ${ }^{105}$

These results highlight the role of time in the emergence of autosuggestibility. Especially after a delay, memories of events may become inaccurate even without outside interference. ${ }^{106}$ For example, in a study by Debra Poole and Lawrence White, four-, six-, and eight-year-old children and adults experienced an event and were repeatedly neutrally questioned about it one week later and then again two years later. ${ }^{107}$ When examining the total amount of inaccurate information reported in response to open-ended questions, they found no age differences after a week-children averaged seven percent inaccurate information compared to six percent for the adults. ${ }^{108}$ After two years, however, children were much more likely than adults to report inaccurate information with error rates of twenty percent versus seven percent, respectively. ${ }^{109}$ Children's inaccuracies ranged from fairly minor, such as incorrect physical descriptions of

97. Id. at 71 .

98. See Lindberg, Interactive, supra note 7, at 52.

99. Id.

100. Stephen J. Ceci et al., Children's Long-Term Memory for Information that is Incongruous with their Prior Knowledge, 72 BRIT. J. PSYCHOL. 443, 444-45 (1981).

101. Id. at 445 .

102. Id. at 448 .

103. Id. at 449 .

104. Id.

105. Id.

106. Debra A. Poole \& Lawrence T. White, Two Years Later: Effects of Question Repetition and Retention Interval on the Eyewitness Testimony of Children and Adults, 29 DEVELOPMENTAL PSYCHOL. 844, 845-49 (1993) [hereinafter Poole, Two Years Later].

107. Id. at 846 .

108. Id. at 848 .

109. Id. at 849 . 
the persons involved in the event, to major, where the actions of one person were attributed to the other. ${ }^{110}$

Suggestions outside of the formal interview context, just like those within, take a variety of forms. Suggestions can come from parents, from other children, and even from within children themselves, and can range from completely false, nonexperienced events to distortions of experienced events. Very little is known about suggestions outside the interview setting because of a myriad of practical and ethical difficulties in studying them. One issue that has received no research attention is the effect of suggesting to a child that a real event never happened. Such a suggestion could be quite different from threats not to disclose experienced events or encouragement to keep actual events secret, because in both of the latter cases the events themselves are not denied. Although researching all types of outside-interview suggestions is difficult, suggestions involving denials of true events could prove especially problematic from an ethical standpoint.

D. It Is Difficult to Identify Particular Children Most Susceptible to Suggestion

In studies such as that by Leichtman and Ceci, ${ }^{111}$ where a sizable minority or, in some cases, a majority of children are led to allege events that did not occur, researchers and practitioners alike have been divided on the meaning and practical implications of the results. ${ }^{112}$ Should we focus on the substantial proportion of children who succumbed to suggestive influences or on those who somehow stood their ground and resisted the repeated and combined forces of various kinds of suggestions from an authoritative adult? Which type better represents the average child? Is there any way to predict whether any individual child witness fits into one category or the other?

In an effort to answer these questions, scores of studies have been conducted over the past decade to investigate relations between suggestibility and various cognitive, personality, and social measures, as well as bio-psycho-social factors such as gender and ethnicity. ${ }^{113}$ Some of the many social and personality factors examined in relation to suggestibility in recent studies include: compliance, dependence, self-esteem, temperament-specifically inhibition and adaptability-and parents' and children's attachment styles-categories of parent-child relationship security. ${ }^{114}$ Some connections between cognitive factors and susceptibility to suggestion seem fairly obvious, including intelligence, memory ability, event-relevant knowledge levels, and source-monitoring abil-

110. Id.

111. Leichtman \& Ceci, supra note 87.

112. Id. at 569 .

113. Many of these studies have been summarized in two recent reviews of the research into individual differences in children's suggestibility. Maggie Bruck et al., External and Internal Sources of Variation in the Creation of False Reports in Children, 9 LEARNING \& INDIVIDUAL DIFFERENCES 289, 305-10 (1997); Jodi A. Quas et al., Individual Differences in Children's and Adults' Suggestibility and False Event Memory, 9 LEARNING \& INDIVIDUAL DIFFERENCES 359, 363-74 (1997).

114. Bruck et al., supra note 113, at 308-10; Quas et al., supra note 113, at 369-72. 
ity. ${ }^{115}$ Children who cannot source-monitor well may not be able to keep track of which pieces of information came from their own experience of the original event and which came from external sources. ${ }^{116}$ Children with greater eventrelevant knowledge, on the other hand, may show increased or decreased suggestibility relative to children who possess less event-relevant knowledge. ${ }^{117} \mathrm{Al}-$ though they may remember more accurate information, children who know more about the theme of an event may infer information that was not presented. ${ }^{118}$

Despite, or perhaps because of, the ever-growing list of factors associated with susceptibility to suggestion, there are several major problems for those who hope to develop diagnostic tests of an individual child witness's suggestibility. First, the single best predictor of suggestibility in children remains their chronological age. ${ }^{119}$ Age is a better predictor because it encompasses development in so many relevant areas, such as increased moral maturity, increased memory monitoring ability, and decreased social compliance with authority. Second, the research on individual differences in children's suggestibility is replete with inconsistent and often weak findings. ${ }^{120}$ For example, intelligence is sometimes related to suggestibility, and other times not. ${ }^{121}$ Finally, there are different forms of suggestion, ${ }^{122}$ and a child's performance on even a single type of suggestibility task varies as a result. ${ }^{123}$ In other words, not only is it difficult to predict a child's suggestibility from a measure of cognitive ability or personality, it is also difficult to predict that child's performance on one suggestibility measure from another suggestibility measure.

Expanding on this latter point, Johann Endres points out that suggestibility in one context may not relate to suggestibility in others because suggestibility is a multidimensional construct and varies depending on memory strength for that specific content, type of suggestion, and the like. ${ }^{124}$ Thus, even a very suggestible child may be able to resist certain misleading questions. Likewise, the fact that a child yields to any given misleading question does not allow inferences about his performance on other questions.

In one recent study by Michael Brady and colleagues, this lack of predictability from one question to another was empirically demonstrated. ${ }^{125}$ Children

115. Bruck et al., supra note 113, at 305-08; Quas et al., supra note 113, at 365-66.

116. Quas et al., supra note 113 , at 365-66.

117. Id. at 367-68.

118. Lindberg, Interactive, supra note 7, at 54.

119. Lane Geddie et al., Child Characteristics Which Impact Accuracy of Recall and Suggestibility in Preschoolers: Is Age the Best Predictor?, 24 CHILD ABUSE \& NEGLECT 223, 230-31 (2000).

120. Quas et al., supra note 113, at 359-60.

121. Bruck et al., supra note 113, at 305.

122. See discussion supra Section Part II.

123. Johann Endres, The Suggestibility of the Child Witness: The Role of Individual Differences and Their Assessment, 1 J. CREDIBILITY ASSESSMENT \& WITNESS PSYCHOL. 44, 52 (1997).

124. Id.

125. Michael S. Brady et al., Young Children's Responses to Yes-No Questions: Patterns and Problems, 3 APPLIED DEVELOPMENTAL SCI. 47, 54-55 (1999). 
between the ages of three and seven years were shown a videotaped event. ${ }^{126}$ Afterwards, they were asked several yes or no questions about the video and then two "suggestibility check" questions unrelated to the videotaped event, including one regarding the name of the interviewer's dog. ${ }^{127}$ Children's performances on these suggestibility check questions had absolutely no relationship to their actual performance on target event questions. ${ }^{128}$

Research on individual differences has revealed numerous variables that may affect children's susceptibility to suggestion. The practical implications of these findings remain unclear. The optimistic position, as described by Jodi Quas and her colleagues, is that identification of the individual difference factors that predict suggestibility may eventually allow legal professionals to take special precautions when interviewing children who are at the greatest risk of incorporating suggestions into their reports. ${ }^{129}$ But individual difference research cannot now, and perhaps never should be, used "retrospectively to indicate whether a particular child's report is or is not true, or as a litmus test to determine whether a given child is even suitable to be a witness."130

E. It Is Difficult to Train Children to Resist Potentially Suggestive Questions or to "Gate-Out" Previously Suggested Information

Given the previously documented difficulties in identifying children who are especially susceptible to suggestive questioning, one of the next logical steps is to teach all children to resist suggestions that they may encounter in the future or to separate prior suggestions from original memories of experienced events. A good deal of research has been dedicated to inducing resistance to suggestion in children, ${ }^{131}$ but less attention has been paid to undoing the damage of suggestions already provided. ${ }^{132}$

One of the earliest studies in which researchers attempted to buffer children against suggestive questioning presented a story to seven-year-olds, twelveyear-olds, and college students, and asked them to recall it. ${ }^{133}$ Half of the participants were then told that they were about to be asked questions, some of which were potentially difficult or tricky, and that they should "only answer

126. Id. at 50 .

127. Id. at 51.

128. Id. at 54 .

129. Quas et al., supra note 113 , at 382.

130. Id.

131. Johann Endres et al., Repetitions, Warnings and Video: Cognitive and Motivational Components in Preschool Children's Suggestibility, 4 LEgAL \& CRIMINOLOGICAL PSYCHOL. 129, 129-46 (1999); Susan Gee et al., "What Color is Your Pet Dinosaur?": The Impact of Pre-interviewing Training and Question Type on Children's Answers, 4 LEGAL \& CRIMINOLOGICAL PSYCHOL. 111 (1999); Amye R. Warren et al., Inducing Resistance to Suggestibility in Children, 15 LAW \& HUM. BEHAV. 273 (1991) [hereinafter Warren, Resistance].

132. Karen J. Saywitz \& Susan Moan-Hardie, Reducing the Potential for Distortion of Childhood Memories, 3 Conscious \& COGNITION 245, 245-66 (1994).

133. Warren, Inducing Resistance, supra note 131, at 276-79. 
with what they really remembered." ${ }^{134}$ This warning was equally effective in reducing suggestibility across the age range, but the reduction, in comparison to the control group, was small at approximately five percent. ${ }^{135}$

Other recent studies have utilized warnings or preinterview training to reduce children's suggestibility. ${ }^{136}$ In one study, a story was presented to fourthrough seven-year-old children. ${ }^{137}$ The story was followed by free recall and then, for half of the children, by an explicit warning about tricky questions. ${ }^{138}$ This warning included a specific example of a question to which the children could not know the answer because the information was not provided in the story, along with the correct answer-"I don't know." ${ }^{139}$ This explicit warning reduced the children's suggestibility by about twelve percent. ${ }^{140}$

Somewhat older children were shown to profit from pre-interview training in a series of two studies. ${ }^{141}$ In the first, children between the ages of nine and thirteen went on a school field trip to an interactive science center. ${ }^{142}$ Approximately a week later, children were interviewed about the trip, and some of the interview questions were misleading. ${ }^{143}$ Before conducting the interviews, half of the children were trained to resist suggestive questions. ${ }^{144}$ The training included instructions not to guess or make up answers, to respond "I don't know" or to correct the interviewer's mistakes when appropriate, and practice with several misleading questions and feedback on answers. ${ }^{145}$ Pre-interview training reduced suggestibility by thirteen percent. It also, however, reduced correct answers to nonleading questions by increasing "don't know" responses. ${ }^{146} \mathrm{~A}$ second study was conducted in which the pre-interview training was revised to emphasize and practice providing correct answers when known. ${ }^{147}$ The revision appeared to be successful in reducing suggestibility by eighteen percent without reducing correct answers for the nine- to eleven-year-olds who were trained prior to the interviews. ${ }^{148}$

The most successful training program for reducing suggestibility was developed by Karen Saywitz and Susan Moan-Hardie. ${ }^{149}$ Seven-year-old children

134. Id. at 278 .

135. Id. at $280,282-83$.

136. Endres et al., supra note 131, at 134; Gee et al., supra note 131, at 115.

137. Endres et al., supra note 131, at 133-35.

138. Id. at 133-34.

139. Id. at 134 .

140. Id. at 135 .

141. Gee et al., supra note 131, at 123-24.

142. Id. at 115 .

143. Id.

144. Id. at 115-16.

145. Id.

146. Id. at 117-20.

147. Id. at 120 .

148. Id. at 122 .

149. Saywitz \& Moan-Hardie, supra note 132. 
witnessed an event in their classrooms. ${ }^{150}$ Two weeks later, half of the children were given extensive "resistance" training, whereas the remaining children were encouraged to try hard and to do their best. ${ }^{151}$ The resistance training involved several components above and beyond those used in the training study described earlier. ${ }^{152}$ For example, children were engaged in discussions about reasons a child might go along with an adult interviewer's false suggestion and were taught new response strategies such as stopping and thinking before answering. Other response strategies included using self-statements ${ }^{153}$ to promote confidence in their own memories and to suppress inappropriate responses. ${ }^{154}$ They also practiced their new skills by watching a video and being interviewed about it afterwards; feedback was provided for their responses to each question. ${ }^{155}$ A review session was conducted the following day, prior to the target interview. ${ }^{156}$ The reduction in suggestibility due to training, compared to the control group, was twenty-six percent. ${ }^{157}$ Unfortunately, as in the prior study, there was a corresponding reduction in correct responses to correctly leading questions. ${ }^{158}$ Thus, a second study was conducted with revised training procedures, placing less emphasis on "don't know" responses and providing more practice and reinforcement in reporting the correct answer when known. ${ }^{159}$ The revised training package resulted in a significant reduction in suggestibility without decreasing accuracy on correctly leading questions. ${ }^{160}$

Despite these encouraging results, it would be premature to declare preinterview training a panacea for the problem of children's suggestibility for several reasons. First, none of these training packages has been attempted, let alone succeeded, in children younger than seven years old, the age group that presents the biggest challenge..$^{161}$ Second, although suggestibility was reduced, it was by no means eliminated. ${ }^{162}$ Third, these training packages must be carefully devised in order to ensure their effectiveness in reducing suggestibility without also decreasing accurate and complete reporting to nonleading questions. ${ }^{163}$ It remains to be determined whether it would be possible or feasible for practitioners to conduct these kinds of extensive and carefully crafted training interventions prior to investigative interviews. Finally, these sorts of interventions have

150. Id. at 414-16.

151. Id. at 415 .

152. Id.

153. Examples of self-statements include: "I know it did not happen like that," "I remember it a different way," and "I should say what I remember." Id.

154. Id.

155. Id.

156. Id.

157. Id. at $416-18$.

158. Id.

159. Id.

160. Id.

161. Gee et al., supra note 131, at 115, 121; Saywitz \& Moan-Hardie, supra note 132, at 251, 255.

162. Gee et al., supra note 131, at 125; Saywitz \& Moan-Hardie, supra note 132, at 258.

163. Gee et al., supra note 131, at 125; Saywitz \& Moan-Hardie, supra note 132, at 258. 
been designed to inoculate children against future suggestions, but children may have already encountered suggestions prior to their first formal forensic interview. ${ }^{164}$ Thus, recent studies have begun to investigate the possibility of helping children to separate experienced from suggested information. ${ }^{165}$

One such study was reported by Debra Poole and Stephen Lindsay. ${ }^{166}$ In a follow-up to their Mr. Science study, ${ }^{167}$ children experienced an event and were later given misinformation about it from a book their parents read to them. ${ }^{168}$ Prior to a final interview, children were trained to "source-monitor," that is, to separate what they had experienced (personal experience source) from what they had only heard about (other source). ${ }^{169}$ This training was successful with six- to eight-year-old children, in that they now correctly attributed some of the false information they had previously reported to the book they had heard rather than personal experience. ${ }^{170}$ However, there was no evidence that the training was effective in reducing reports of nonexperienced events in three- to five-year-olds. ${ }^{171}$

A different technique for reducing the effects of previously suggested false information was studied by Mary Lyn Huffman and colleagues. ${ }^{172}$ Children between the ages of four and six years participated in an event at their preschools with a visitor named Sam Stone and were interviewed a week later. ${ }^{173}$ Some of the interview questions involved nonexperienced events whereas others embedded misleading or correctly leading information about actually experienced events. ${ }^{174}$ Two days following this interview, children were reinterviewed with specific but nonleading questions. ${ }^{175}$ These final interviews began in one of three different ways. ${ }^{176}$ One-third of the children were first asked three questions about the difference between truth and lying. ${ }^{177}$ Another third were asked

164. Poole \& Lindsay, Eyewitness, supra note 74, at 27.

165. Brett K. Hayes \& Katrina Delamothe, Cognitive Interview Procedures and Suggestibility in Children's Recall, 82 J. APPLIED PSYCHOL. 562 (1997); Mary Lyn Huffman et al., Discussing Truth and Lies in Interviews Children: Whether, Why, and How?, 3 APPLIED DeVElopMENTAL SCI. 6 (1999); Amina Memon et al., Reducing Suggestibility in Child Witness Interviews, 10 APPLIED CogNitive PSYCHOL. 503 (1996); Susan M. Larson, Truth/Lie Discussions and Preschoolers' Memories of Personally Experienced Events (1999) (unpublished master's thesis, University of Tennessee at Chattanooga) (on file with the University of Tennessee at Chattanooga Library); Debra A. Poole \& D. Stephen Lindsay, Counteracting the Effects of Contamination on Children's Eyewitness Reports, Eliciting Accurate Testimony from Children: What Works, What Doesn't, and Why?, A Symposium presented at the Biennial Meeting of the Society for Research in Child Development, Amye R. Warren \& Debra A.

Poole, Chairs (April 1999) [hereinafter Poole \& Lindsay, Effects].

166. Poole \& Lindsay, Effects, supra note 165.

167. Poole \& Lindsay, Eyewitness, supra note 74.

168. Poole \& Lindsay, Effects, supra note 165, at 1.

169. Id. at 2 .

170. Id.

171. Id.

172. Huffman et al., supra note 165 , at 8 .

173. Id. at 11 .

174. Id.

175. Id. at 12 .

176. Id.

177. Id. 
many more elaborate questions about truth and lying and were asked to judge three vignettes about other children telling the truth or lying. ${ }^{178}$ The final group of children did not discuss truth or lying before the questioning began. ${ }^{179}$ All children were asked to tell the truth about Sam Stone's visit. ${ }^{180}$ Children who had been asked the lengthiest series of questions about truth and lying were significantly more accurate in their responses to the final neutral interview than were the other two groups of children. ${ }^{181}$ A follow-up study by one of the researchers, however, failed to replicate this effect. ${ }^{182}$

Another method for reducing contamination from prior suggestions was investigated by two research groups with different outcomes. ${ }^{183}$ In both studies, either the Cognitive Interview ${ }^{184}$ or a standard interview was conducted after exposure to misleading questions. ${ }^{185}$ In one study, eight- to nine-year-old children witnessed a videotaped event and were then asked misleading questions. ${ }^{186}$ Next, they were reinterviewed with either the cognitive or standard interview, and then another set of misleading questions was administered. ${ }^{187}$ Relative to a standard interview, the cognitive interview increased accuracy and decreased false answers to subsequent misleading questions. ${ }^{188}$ However, one-third of all children reported false information even in free recall, and suggestibility appeared to increase with the second set of misleading questions. ${ }^{189}$ Thus, the cognitive interview did not reduce suggestibility in any absolute way, but only relative to the standard interview. ${ }^{190}$ In a second study examining the potential of the cognitive interview for reducing children's suggestibility, five- to seven-yearold and nine- to eleven-year-old children were first exposed to a videotaped event and then to misleading information about it, followed three days later by either the cognitive or standard interview. ${ }^{191}$ The cognitive interview did not prove to be superior to a standard interview in terms of children's tendency to intrude suggested answers in free recall and answers to direct questions. ${ }^{192}$

178. $I d$.

179. $I d$.

180. Id.

181. Id. at 13 .

182. Larson, supra note 165.

183. Hayes \& Delmothe, supra note 165, at 564, 568-71; Memon, supra note 165, at 507-08, 510-13.

184. Ronald P. Fisher \& R. EdWARd GEISElman, MEMORY ENHANCING TeCHNiQues fOR InVESTIGATIVE INTERVIEWING: THE COGNITIVE INTERVIEW 41, 100, 110-11(1992). The Cognitive Interview was designed, based on findings from memory research, to enhance the completeness and accuracy of eyewitness accounts. The Cognitive Interview includes special mnemonic techniques and instructions to report everything the witness remembers. $I d$.

185. Hayes \& Delmothe, supra note 165, at 565-67; Memon, supra note 165, at 508-09.

186. Memon, supra note 165 , at 508-09.

187. Id.

188. Id. at 510.

189. Id. at 511-13.

190. Id. at 510-13.

191. Hayes \& Delmothe, supra note 165 , at 565-67.

192. Id. at 568-70. 
Finally, Michelle Leichtman and Stephen Ceci "challenged" children who had reported suggested information with "Did you really see that or just hear about it?" and a countersuggestion, "You didn't really see him do that, did you?" "193 In their most suggestive condition involving repeated misleading questioning plus the induction of a negative stereotype, initially seventy-two percent of the children responded falsely to a direct question about Sam Stone's behavior. ${ }^{194}$ With the first challenge, this rate dropped to forty-four percent, and with the countersuggestion it fell further, to twenty-one percent. ${ }^{195}$ Thus, these challenges were effective in eliminating two-thirds of the false allegations. ${ }^{196}$

A growing literature is documenting a variety of techniques to reduce the impact of suggestions once they have already occurred. These research efforts are in their infancy, however, and the successes appear inconsistent across studies and age groups. Moreover, as with the techniques designed to increase resistance to suggestions before they occur, these techniques do not eliminate suggestibility, and they may be impractical or unethical for use in the field. For example, if a child reported that someone had inserted a stick into her vagina, it would be completely inappropriate and probably counterproductive to obtaining any further truthful disclosures to say, "That didn't really happen, did it?"

F. It Is Difficult to Train Interviewers to Avoid Suggestive Techniques and to Use Techniques Designed to Promote Accuracy

Given the difficulties of identifying particularly suggestible children and of training children to resist suggestive influences, it is important for interviewers to avoid the use of suggestive techniques. Although this seems obvious, the interviewers studied in many different countries tend to over-use closed-ended, specific, and potentially leading questions and other "risky" practices. ${ }^{197}$ For example, one study examined seventy-two interviews conducted by experienced interviewers in Sweden. ${ }^{198}$ Despite universal recommendations to begin interviews with general, open-ended or "invitational" questions that promote fairly spontaneous, narrative responses, thirty-five of these interviews (forty-nine percent) began with a suggestive question. ${ }^{199}$ Throughout the interviews, the interviewers relied on suggestive and "option-posing" (forced-choice) questions, which accounted for fifty-three percent of the interviewers' utterances, and elicited fifty-seven percent of the information from children. ${ }^{200}$ Similarly, fiftythree percent of the utterances of a comparison sample of United States interviewers and thirty-five percent of Israeli interviewers' utterances were sugges-

193. Leichtman \& Ceci, supra note 87 , at 571.

194. Id. at 573 .

195. Id.

196. $I d$.

197. Ann-Christin Cederbog et al., Investigative Interviews of Child Witnesses in Sweden, 24 CHILD ABUSE \& NEGLECT 1355 (2000).

198. Id. at 1356-57.

199. Id. at $1358-59$.

200. Id. at 1359 . 
tive or option-posing. ${ }^{201}$ Only six percent of the Swedish interviewers' utterances were invitational. ${ }^{202}$ Corresponding figures for Israeli and United States interviewers were similar, at two percent and five percent, indicating remarkable consistency across cultures with different interview selection and training procedures. ${ }^{203}$

Other studies of United States interviewers have documented similar problems. An analysis of forty-two United States sexual abuse interviews found that general, open-ended questions account for ten percent or fewer of all interviewer questions, and that specific, yes-or-no-format questions account for twothirds of all questions. ${ }^{204}$ In addition, interviewers sometimes (twenty-nine percent of the time) completely fail to establish rapport and often (seventy-one percent of the time) fail to establish interview ground rules by telling children that they should feel free to correct the interviewers and to answer that they do not remember or do not understand questions. ${ }^{205}$

Can interviewers' performance be improved such that they reduce their reliance on risky techniques and increase their use of recommended techniques? Several recent training evaluation programs suggest that this is more difficult than it seems. ${ }^{206}$

Jan Aldridge and Sandra Cameron examined the effectiveness of a weeklong intensive interviewer training program in the United Kingdom. ${ }^{207}$ No differences between trained and untrained interviewers were observed in terms of their use of open, specific, and leading questions. ${ }^{208}$ Moreover, there were very few requests for free reports, such as invitational, in either group, and over half of the questions in both groups were specific and leading. ${ }^{209}$

In a similar evaluation study, the effectiveness of a ten-day interviewer training institute was examined by comparing pre- to post-training videotaped interviews with children about previously experienced staged events. ${ }^{210}$ Analyses of the use of open-ended, specific, and leading and misleading questions in-

201. Id.

202. Id.

203. $I d$.

204. Warren et al., Sounds, supra note 59, at 236-37.

205. Id. at 233-34.

206. Jan Aldridge \& Sandra Cameron, Interviewing Child Witnesses: Questioning Techniques and the Role of Training, 3 APPLIED DEVELOPMENTAL SCI. 136 (1999); Graham M. Davies et al., The Impact of Questioning Style on the Content of Investigative Interviews with Suspected Child Sexual Abuse Victims, 6 PSYCHOL. CRIME \& L. 81 (2000); Yael Orbach et al., Assessing the Value of Structured Protocols for Forensic Interviews of Alleged Child Abuse Victims, 24 CHILD ABUSE \& NEGLECT 733 (2000); Amye R. Warren et al., Assessing the Effectiveness of a Training Program for Interviewing Child Witnesses, 3 APPLIED DEVelopmental SCI. 128 (1999) [hereinafter Warren et al., Effectiveness]; Home OFFICE IN CONJUNCTION WITH THE DEPARTMENT OF HEAlTh, MEMORANDum OF GoOD PRACTICE ON VIDEO RECORDED INTERVIEWS WITH CHILD WitNesSES FOR CRIMINAL PROCEEDINGS (1992).

207. Aldridge \& Cameron, supra note 206, at 138.

208. Id. at 142 .

209. Id.

210. Warren et al., Effectiveness, supra note 206, at 129-30. 
dicated no significant changes due to training. ${ }^{211}$ On a more encouraging note, interviewers were significantly more likely to establish the ground rules in the interviews they conducted after training (forty-five percent) than before training (five percent). ${ }^{212}$ Additionally, interviewers' knowledge of the relevant scientific literature increased due to training, even though their new knowledge did not appear to translate directly into new interview practices. ${ }^{213}$

In England, Graham Davies, Helen Westcott, and Noreen Horan assessed interviews conducted by interviewers trained in techniques outlined by the Memorandum of Good Practice. ${ }^{214}$ Thirty-six interviews were examined, most of which had been conducted in the mid- to late 1990s, during which time training was more widely available than before. ${ }^{215}$ Despite the fact that all of the interviewers had been trained in Memorandum-recommended practices, only two percent of their questions were open-ended. ${ }^{216}$ Again, some encouraging results were found as well, in that only three percent of these interviewers' utterances were classified as leading. ${ }^{217}$

The most encouraging findings to date on interviewer training effectiveness were reported by Yael Orbach and her associates. ${ }^{218}$ Their training program differed in many critical ways from those evaluated in the studies discussed previously. ${ }^{219}$ First, they trained interviewers to use a very structured protocol, similar to a "script."220 Thus, rather than being encouraged to use open-ended questioning, interviewers were given sample open-ended questions to ask and the order in which they should be posed. ${ }^{221}$ Second, the initial intensive training was followed with monthly individual sessions and group discussions that included utterance-by-utterance reviews of videotaped interviews. ${ }^{222}$ Interviews conducted by trained interviewers using the protocol were compared to nonprotocol interviews conducted previously by the same interviewers. ${ }^{223}$ The protocol interviews were significantly improved in terms of the percentage of open-ended prompts, as well as how long interviewers waited before asking their first potentially suggestive (option-posing) question. ${ }^{224}$ Protocol interviews also yielded more information from open-ended prompts and less from more specific, focused questions than did non-protocol interviews. ${ }^{225}$ There were,

211. Id. at 132-33.

212. Id. at 133 .

213. Id. at 132 .

214. Davies et al., supra note 206, at 85.

215. Id.

216. Id. at 86 .

217. Id.

218. Orbach et al., supra note 206, at 737.

219. Id. at 737-40.

220. Id. at 739 .

221. Id. at 738-39.

222. Id.

223. Id. at 737 .

224. Id. at 741 .

225. Id. at 741-43. 
however, no differences between protocol and non-protocol interviews in terms of the total amount of abuse-relevant information elicited. ${ }^{226}$

Extant research thus demonstrates that interviewer training is effective in reducing problematic questioning techniques only when training is both intensive and extensive, and only when it includes practice, individualized feedback, and follow-up. ${ }^{227}$ Across the United States, interviewer guidelines vary dramatically, and there is little documentation of current interview practices. $^{228}$ Improving the quality of investigative interviews with children will, therefore, require greater commitment of and cooperation between researchers and practitioners, and far greater investments of effort and resources on the part of the research and legal communities alike.

III

\section{CONCLUSION}

In this volume, many psycho-legal and legal scholars have attempted to capture and describe the state of our current knowledge of children's testimony. Certainly a great deal has been learned about children's strengths, weaknesses, and needs as legal witnesses. This paper has instead focused on six topics about which we know relatively little: suggestibility in older children, suggestions outside of leading questions and outside formal interview settings, individual differences in suggestibility, and methods for reducing children's susceptibility to suggestive techniques and interviewers' tendencies to use them. If the growth in understanding of children's testimony over the last twenty years is any indication of the gains we will make over the next twenty, many of these six dilemmas may soon be resolved.

226. Id. at $745-46$.

227. Davies et al., supra note 206, at 93-94; Warren et al., Effectiveness, supra note 206, at 134-35.

228. Lucy S. McGough, Good Enough for Government Work: The Constitutional Duty to Preserve Forensic Interviews of Child Victims, 65 LAW \& CONTEMP. PROBS. 179 (Winter 2002); Nancy E. Walker, Forensic Interviews of Children: The Components of Scientific Validity and Legal Admissibility, 65 LAW \& CONTEMP. PROBS. 149 (Winter 2002). 